\title{
Persistent infection of betanodavirus in a novel cell line derived from the brain tissue of barramundi Lates calcarifer
}

\author{
S. C. Chi*, Y. C. Wu, T. M. Cheng \\ Department of Life Science and Institute of Zoology, National Taiwan University, 1, Sec. 4, Roosevelt Rd., Taipei 10617, Taiwan
}

\begin{abstract}
In order to obtain an in vitro system for studying the mechanism of persistent infection of fish nodavirus, a novel cell line (BB) was established from the brain tissue of a barramundi, Lates calcarifer, which had survived viral nervous necrosis disease. The cell line has been subcultured $>100$ times. The persistence of fish nodavirus designated as barramundi brain nervous necrosis virus (BBNNV) in the BB cells was demonstrated by: (1) the detection of the infectious virus in the culture supernatants, (2) the detection of NNV nucleic acids extracted from the BB cells, (3) the positive result of immunochemical staining using an NNV-specific monoclonal antibody and (4) their resistance to infection by another fish nodavirus grouper NNV (GNNV). No temperature-sensitive mutants were detected in the culture supernatant of the BB cells. Neither truncated genome (RNA1 or RNA2) nor smaller coat protein was found in the purified BBNNV particles, suggesting that defective interfering particles were unlikely to be important in the NNV-persistent infection in the BB cells. The result of the neutralization test indicated that the 5 antigenic determinants, recognized by GNNV-specific neutralizing antibodies, also existed on the coat protein of BBNNV. The BB cell line is the first cell line reported to be persistently infected with NNV, and would be a useful model for understanding the mechanisms of NNV-persistent infection in vitro and in vivo.
\end{abstract}

KEY WORDS: Fish cell line · Persistent infection · Betanodavirus · Viral nervous necrosis

\section{INTRODUCTION}

Fish nodavirus designated as nervous necrosis virus (NNV) is the causative agent of viral nervous necrosis (VNN) disease, and has caused mass mortality of various species of fish at the larval and juvenile stages (Munday et al. 2002, Chi et al. 2003). The infected fish display abnormal swimming behavior, and the most common histopathological change observed in NNV-infected fish is the vacuolation of the brain and retina. The NNV is a non-enveloped, icosahedral virion (25 to $30 \mathrm{~nm}$ ), and has been identified as a fish nodavirus (or betanodavirus) of Nodaviridae, with 2 segmented, single-stranded, positive sense RNAs (Mori et al. 1992, Comps et al. 1994, Chi et al. 2001a). The RNA1 encodes an RNA-dependent RNA polymerase of approximately $100 \mathrm{kDa}$, and the RNA2 encodes a coat protein of approximately $42 \mathrm{kDa}$. Fish nodaviruses are classified into 4 genotypes based on the nucleotide sequences of the coat protein genebarfin flounder (BF) type, tiger puffer (TP) type, striped jack (SJ) type and red-spotted grouper (RG) type (Nishizawa et al. 1997). The RGNNV genotype has the widest host spectrum of the 4 genotypes (Munday et al. 2002). In Taiwan, NNV was first detected in hatchery-reared grouper (Chi et al. 1997), and has since been found in 8 other species of cultured fish (Chi et al. 2003). All Taiwan NNV isolates belong to the RGNNV genotype.

Fish nodavirus has been re-isolated from the persistently infected wolfish Anarhichas minor by SSN-1 cells 16 wk post-bath-challenge (Johansen et al. 2003). However, the information about the carrier-state of survivor fish remains very limited, and studies on the 
mechanisms of NNV-persistent infection are absent. Persistent infections in cell cultures have been established with members of most of the major RNA virus groups (Rima \& Martin 1976, Hedrick et al. 1978). All of these persistently infected cell cultures share the following characteristics: (1) continued presence of a viral antigen in the majority of cells, (2) continued production of the infectious virus, (3) resistance to superinfection by a homologous virus and (4) continued cell division and growth of carrier cells, which are indistinguishable from normal, uninfected cells. Persistent infection results from continuous production of the infectious virus; this is achieved either by survival of infected cells or by a situation in which the minority of cells are initially infected and the spread of the virus is limited, so that cell death is counterbalanced by new cells produced by division, i.e. no net loss (Dimmock \& Primrose 1994). Examinations of persistently infected cells have revealed that the mechanisms that control viral persistence in vitro may also operate during infections in the intact host (Hedrick \& Fryer 1981).

In the present study, we developed a novel cell line (BB) from the brain tissue of a barramundi that had survived VNN disease, and we identified the persistence of NNV infection in this cell line. We also examined whether temperature mutants or defectiveinterfering (DI) particles existed in the BB cell line.

\section{MATERIALS AND METHODS}

Cells and viruses. The GF-1 cell line from grouper fin (Chi et al. 1999) was propagated in Leibovitz's L-15 medium supplemented either with $5 \%$ fetal bovine serum (FBS) for routine subcultures or $2 \%$ for virus amplification and titration.

GNNV (G9508KS) isolated from grouper larvae with VNN disease (Chi et al. 2001a) was applied for the superinfection of BB cells. Another Taiwan isolate, B00GD, derived from barramundi larvae during the acute stage of VNN disease (Chi et al. 2003), was used to compare the antigenecity, genomic RNA profile and protein profile with the virus isolate BBNNV from the BB cell line.

Fish samples. Survivors of cultured barramundis, Lates calcarifer, with body lengths of 7 to $8 \mathrm{~cm}$, were collected 1 mo after VNN disease was detected in southern Taiwan. The nucleic acids of the NNV coatprotein gene were detected in 5 of the surviving fish by semi-nested PCR using NNV-specific primers. One of the remaining surviving fish was used for primary culture.

Development of the BB cell line. Primary culture and subculture were performed according to the method described by Chi et al. (1999). Briefly, the fish was first wiped with $70 \%$ alcohol. The brain tissue was removed by dissection and washed 3 times in a washing buffer (phosphate-buffered saline, PBS, containing $400 \mathrm{IU}$ penicillin $\mathrm{ml}^{-1}, 400 \mu \mathrm{g}$ streptomycin $\mathrm{ml}^{-1}, 1 \mu \mathrm{g}$ amphotericin $\mathrm{ml}^{-1}$ ). After initial washing, the tissue was minced with scissors and washed 3 times in a washing medium (L-15 medium containing $400 \mathrm{IU}$ penicillin $\mathrm{ml}^{-1}, 400 \mu \mathrm{g}$ streptomycin $\mathrm{ml}^{-1}, 1 \mu \mathrm{g}$ amphotericin $\mathrm{ml}^{-1}$ ). The tissue fragments were then transferred into $25 \mathrm{~cm}^{2}$ tissue culture flasks, supplemented with complete culture medium (L15 plus $20 \%$ FBS, $100 \mathrm{IU}$ penicillin $\mathrm{ml}^{-1}, 100 \mu \mathrm{g}$ streptomycin $\mathrm{ml}^{-1}$ and $0.25 \mu \mathrm{g}$ amphotericin $\mathrm{ml}^{-1}$, and incubated at $28^{\circ} \mathrm{C}$. When a confluent monolayer was formed, cells were dislodged by trypsin-EDTA solution $(0.1 \%$ trypsin and $0.2 \%$ EDTA in PBS), and then split at a 1:2 ratio into new flasks after adding fresh culture medium.

The cell type of the BB cell line was determined by immunostaining using mouse monoclonal antibodies (MAbs) against 3 kinds of cell markers (NeoMarkers, Lab Vision): (1) neurofilament Ab-1 (Clone 2F11) against the intermediate filaments of neurons and their process, (2) glial fibrillary acidic protein Ab-3 (Clone GA-5 + 6F2) against the glial fibrillary acidic protein (GFAP) in astrocyte, glial cells and ependymal cells and (3) keratin Pan Ab-2 (PAN-CK cocktail) against a broad spectrum of keratins in epithelial tumors. The cells were fixed with $10 \%$ formalin, incubated with $0.2 \%$ Triton-X 100 for several minutes, washed with PBST (phosphate buffer with $0.05 \%$ Tween 20), blocked with $3 \%$ skimmed milk, reacted with cell marker-specific MAb (1:500 diluted in PBST) at room temperature for $1 \mathrm{~h}$ and stained by FITC-conjugated goat antimouse antibodies. The GF-1 cell line was also immunostained by these 3 MAbs as a positive control for epithelial cells.

Identification of the persistence of virus in the $\mathrm{BB}$ cell line. In order to determine whether viral persistence exists in the BB cell line, culture supernatant of BB cells was collected and filtered through a $0.2 \mu \mathrm{m}$ Millipore membrane. The filtrate was then inoculated into a monolayer of GF-1 cells, and the cytopathic effect (CPE) was checked after $6 \mathrm{~d}$ of incubation at $28^{\circ} \mathrm{C}$.

Detection of NNV in BB cells by RT-PCR and seminested PCR. RT-PCR and semi-nested PCR were used to detect the existence of NNV nucleic acids in BB cells according to the procedures described by Lee et al. (2002). The T2 and T4 regions of the coat protein gene of NNV were amplified using the primer sets F1/R3 and F2/R3, respectively. Primer sequences of F1 (5'GGATTTGGACGTGCGACCAA-3'), F2 (5'-CGTGTCAGTGCTGTGTCGCT-3') and R3 (5'-CGAGTCAACACGGGTGAAGA-3') were based on those described 
by Nishizawa et al. (1994). Amplification was performed by an initial denaturation of $3 \mathrm{~min}$ at $94^{\circ} \mathrm{C}$, followed by 30 cycles of $30 \mathrm{~s}$ at $94^{\circ} \mathrm{C}, 30 \mathrm{~s}$ at $60^{\circ} \mathrm{C}$ and $45 \mathrm{~s}$ at $72^{\circ} \mathrm{C}$, with a final extension of $5 \mathrm{~min}$ at $72^{\circ} \mathrm{C}$. The expected sizes of the T2 and T4 regions are 864 and $414 \mathrm{bp}$, respectively.

Detection of NNV in BB cells by immunochemical staining. Immunochemical staining using GNNVspecific MAb 9D (Chi et al. 2001b) was applied to detect the NNV protein in $\mathrm{BB}$ cells and the ratio of NNV-infected cells in the BB cell line. The cells were seeded in $30 \mathrm{~mm}$ petri dishes supplemented with culture medium, and grown to $70-80 \%$ confluence at $28^{\circ} \mathrm{C}$. The cells were fixed with $10 \%$ formaldehyde for 10 min and blocked with $5 \%$ bovine serum albumin (BSA) in Tris buffer solution (TBS) for $15 \mathrm{~min}$. After incubation with GNNV-specific MAb 9D (1:1000 diluted in TBS) for $1 \mathrm{~h}$, the cells were stained according to the procedures of the UltraTech AP streptavidinbiotin universal detection system (Immunotech). Briefly, the cells were washed with TBS 3 times, covered with biotinylated secondary antibody for 15 min, then washed by another 3 changes of TBS and, finally, reacted with streptavidin-alkaline phosphatase reagent for $15 \mathrm{~min}$. Chromogen development was done with freshly prepared Fast Red working solution for 15 min. Hematoxylin was used as a counterstain. All procedures were done at room temperature.

Superinfection by another betanodavirus. Grouper NNV (GNNV), another fish nodavirus of the RGNNV genotype, was used to identify the resistance of the BB cell line to closely related betanodavirus superinfection. The multiplicity of infection (MOI) was 1. The CPE (cytopathic effect) was examined after $6 \mathrm{~d}$ of incubation at $28^{\circ} \mathrm{C}$.

Titration of the virus in the supernatants of BB cells. In order to observe the variation of BBNNV titers from 30 (41 70) subcultures, supernatants were collected $5 \mathrm{~d}$ after each subculture of BB cells and titrated in GF-1 cells. The titration method was similar to the method described by Lee et al. (2002). Viral titers are expressed as TCID $50 \mathrm{ml}^{-1}$.

Temperature effect on the replication of BBNNV. The BB cells were seeded into $25 \mathrm{~cm}^{2}$ flasks $(1 \times$ $10^{6}$ cells per flask), and then incubated at 4 different temperatures $\left(20,25,28\right.$ and $\left.33^{\circ} \mathrm{C}\right)$ for $6 \mathrm{~d}$. The supernatants of cultured cells were collected at the end of incubation and titrated in GF-1 cells at $28^{\circ} \mathrm{C}$.

Amplification and purification of $B B N N V$ and B00GD. Both BBNNV and B00GD were separately propagated in 40 flasks $\left(75 \mathrm{~cm}^{2}\right)$ of GF-1 cells. Then, $3 \mathrm{ml}$ undiluted culture supernatant of BB cells, with a titer of $10^{4} \mathrm{TCID}_{50} \mathrm{ml}^{-1}$, was first inoculated into GF-1 cells, and $6 \mathrm{ml}$ fresh medium was then added after $1 \mathrm{~h}$ viral adsorption. The MOI for B00GD infection was 1.
Complete CPE appeared in both BBNNV-infected and B00GD-infected GF-1 cells 6 d post-infection (p.i.), and the viruses were purified with a procedure described by Chi et al. (2001a).

Analysis of RNA and protein profiles of purified BBNNV and B00GD. The methods for acid guanidinium thiocyanate-phenol-chloroform extraction of genomic RNA from purified virions and for electrophoretic analysis were similar to those described by Chi et al. (2001a).

The protein profiles of BBNNV and B00GD were analyzed by SDS-PAGE and Western blot. Purified B00GD and BBNNV were adjusted to the same concentration, and applied for $10 \%$ SDS-polyacrylamide gel electrophoresis (PAGE). After SDS-PAGE, proteins were eletrophoretically transferred to a PVDF membrane (Millipore). The membrane was washed in TBS buffer and blocked with $5 \%$ skim milk in TBS overnight. After washing, the membrane was reacted with partially purified, GNNV-specific MAb 9D, and then with alkaline-phosphatase (AP)-conjugated goat antimouse Ig antibody (KPL). Finally, the protein bands were visualized by the AP conjugate substrate kit (KPL).

Analysis of the deduced amino acid sequence of BBNNV. Viral RNA was extracted from BBNNV in the culture supernatants of the BB cell line, and amplified by RT-PCR according to the method described by Lee et al. (2002). Automatic sequencing of RT-PCR product T2 of BBNNV was performed commercially (Mission Biotech). Multiple alignments of determined nucleotide and deduced amino acid sequences were constructed with the Lasergene program (DNASTAR). The deduced amino acid sequence of the T2 region of the coat protein gene of BBNNV was compared with the corresponding region of the 9 reported Taiwan NNV isolates, including G9410YA, G9508KS, EE98PH, YP99PD, FS99PD, B00GD, Co00CC1, HG00GD and CC01YL1 (Chi et al. 2003). The antigenic index (Jameson \& Wolf 1988), a computer algorithm that can be used to predict the topological features of a protein directly from its primary amino acid sequence, was also determined by the Lasergene program (DNASTAR).

Neutralization test. Five neutralizing monoclonal antibodies (2E, 9B, 9D, 10G and 12E) against GNNV (Chi et al. 2001b, Shieh \& Chi 2005) were used for the alpha neutralization test (constant antibody plus virus dilution) according to the method described by Chi et al. (2003). The neutralization index (NI) was determined by the difference between the titer of the control and that of the MAb-treated viral samples. The neutralization effect is expressed as $\log \mathrm{NI}$, and is considered to be significant when $\log _{10}$ NI $>1.7$ (Mahy \& Kangro 1996). 


\section{RESULTS}

\section{Characteristics of the BB cell line}

A few swollen and rounded cells that exhibit similar morphology to the characteristic CPE induced by NNV (Chi et al. 1999) have frequently been observed in the BB cell line since primary culture (Fig. 1). This cell line has been subcultured $>100$ times, with a split ratio of 1:2 every 3 to $4 \mathrm{~d}$. The BB cell line is able to grow at temperatures from 20 to $30^{\circ} \mathrm{C}$, but the optimal temperature is $28^{\circ} \mathrm{C}$.

All the BB cells and GF-1 cells showed positive reactions with mouse MAbs against a broad spectrum of keratins in epithelial tumors, but they showed negative results with MAbs against neurofilament or glial fibrillary acidic proteins.

\section{Identification of the NNV-persistent infection in the $\mathrm{BB}$ cell line}

Three days after inoculating the supernatant of BB cells into GF-1 cells, a specific CPE with swollen and rounded cells was observed, and complete cell lysis occurred on the 5th day (Fig. 2). The CPE in the infected GF-1 cells was very similar to the GNNVinduced CPE described in our previous paper (Chi et al. 1999). The titer of the supernatant of the infected GF-1 cells after complete CPE was $10^{9} \mathrm{TCID}_{50} \mathrm{ml}^{-1}$, indicating that infectious virions existed in the supernatant of BB cells and efficiently replicated in GF-1 cells.

The nucleic acid of the betanodavirus coat protein gene could be detected in the infected GF-1 cells by both RT-PCR and semi-nested PCR using NNV-

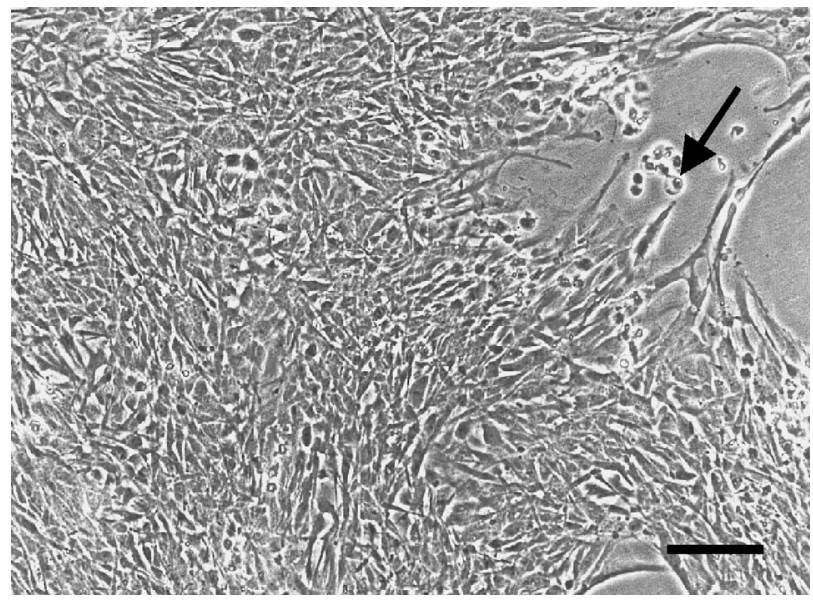

Fig. 1. Morphology of a confluent monolayer of barramundi brain (BB) cells. The arrow indicates the swollen and rounded cells similar to the cells with characteristic cytopathic effect

(CPE) of nervous necrosis virus (NNV). Scale bar $=10 \mu \mathrm{m}$

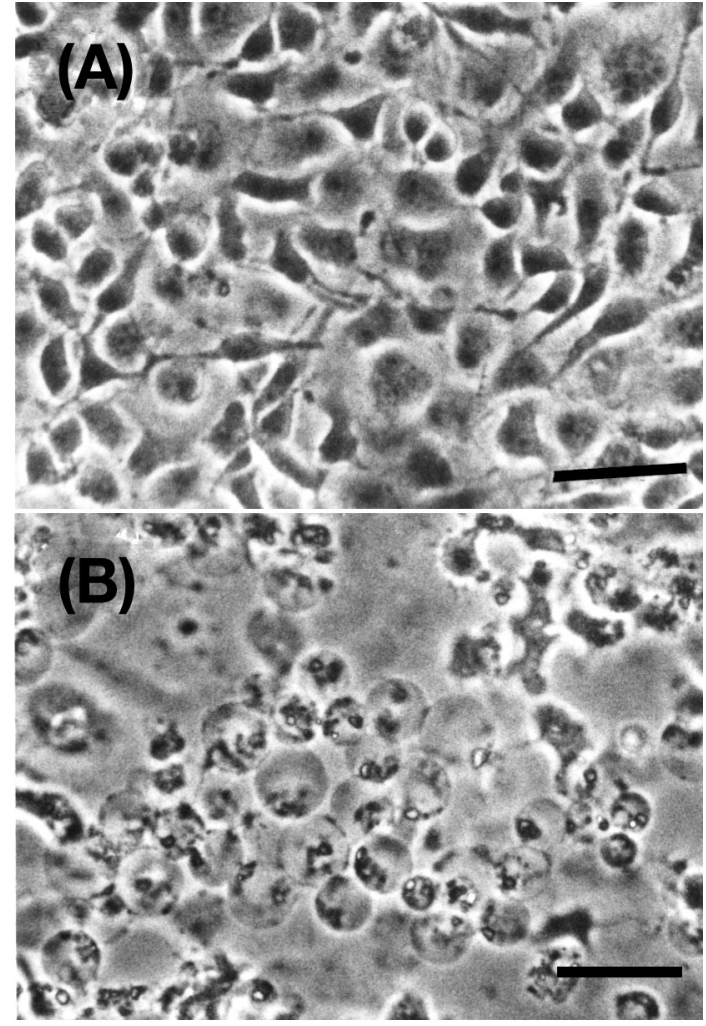

Fig. 2. Morphology of (A) non-infected GF-1 cells and (B) GF-1 cells with characteristic CPE induced by inoculation of the undiluted culture supernatant of BB cells. Scale bars $=50 \mathrm{~nm}$

specific primer sets (Fig. 3), suggesting that the virus causing the CPE of GF-1 cells was a betanodavirus designated BBNNV.

The nucleic acid of the BBNNV coat protein gene was also detected in BB cells, but the amount was below the detection limit of RT-PCR and could only be amplified by semi-nested PCR (Fig. 3B). The cell numbers of BB cells and the infected GF-1 cells were the same; however, the level of BBNNV nucleic acids in BB cells was much lower than that present in the infected GF-1 cells.

Furthermore, only $1 \%$ of the BB cells in the cell population showed a positive reaction with GNNVspecific MAb 9D (Fig. 4), but the strong staining signal suggested that BBNNV could replicate very well in the cytoplasm of these few BB cells.

The supernatants of BB cells were collected $5 \mathrm{~d}$ from each subculture and titrated in GF-1 cells at $28^{\circ} \mathrm{C}$. The titers of BBNNV in Subcultures 41 to 70 of the BB cells varied from $10^{3.2}$ to $10^{6.3} \mathrm{TCID}_{50} \mathrm{ml}^{-1}$ (Fig. 5). Such fluctuation suggested that factors interfering with normal viral replication existed in the BB cell line. In addition, $\mathrm{BB}$ cells resisted the superinfection of another betanodavirus GNNV, and no CPE was observed even $6 \mathrm{~d}$ post-infection. 


\section{Temperature effect on BBNNV amplification}

The BB cells, with same cell density in each flask, were incubated at 4 temperatures for $6 \mathrm{~d}$. Supernatants were then collected and titrated in GF-1 cells at $28^{\circ} \mathrm{C}$. The titers of the supernatants from $\mathrm{BB}$ cells incubating at the temperatures of $20,25,28$ and $33^{\circ} \mathrm{C}$ were $10^{3.0}$, $10^{3.3}, 10^{4.3}$ and $10^{4.0} \mathrm{TCID}_{50} \mathrm{ml}^{-1}$, respectively. No CPE was observed in BB cells cultured at any one of these
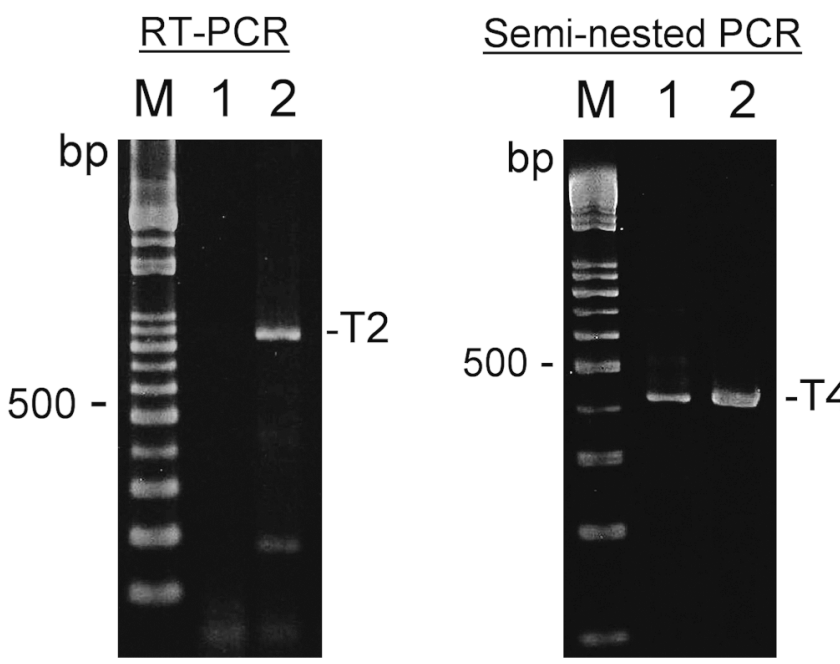

Fig. 3. RT-PCR and semi-nested PCR detection of NNV nucleic acids extracted from BB cells (Lane 1) and BBNNVinfected GF-1 cells $5 \mathrm{~d}$ after inoculation with the supernatant of BB cells (Lane 2). M: DNA marker (100 bp ET ladder); T2: target product of RT-PCR using the primer set F1/R3; T4: target product of semi-nested PCR using the primer set F2/R3

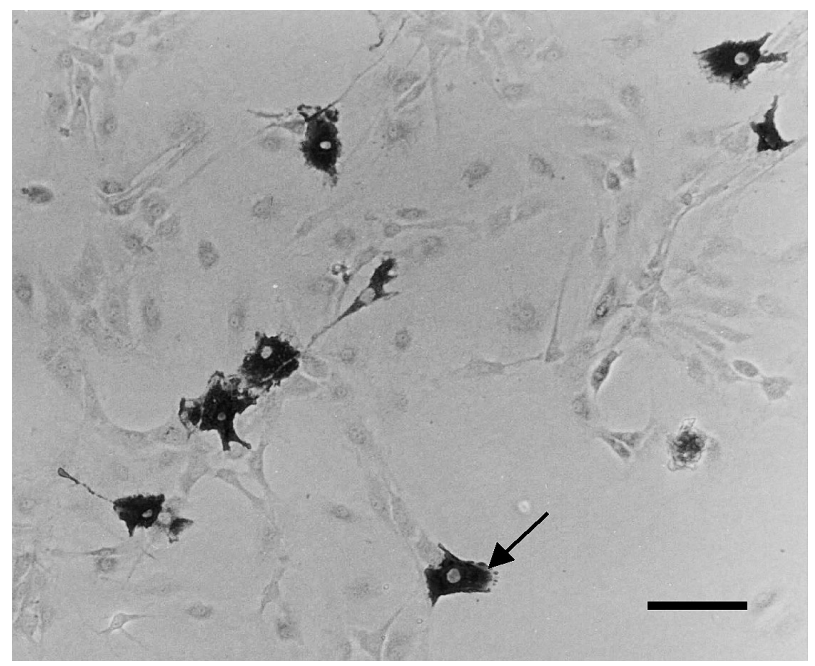

Fig. 4. Detection of NNV-persistently infected BB cells by immunochemical staining using grouper NNV (GNNV)specific MAb 9D (an NNV-specific monoclonal antibody). The arrow indicates a positive signal in the cytoplasm of NNV-infected BB cells. Scale bar $=87 \mu \mathrm{m}$

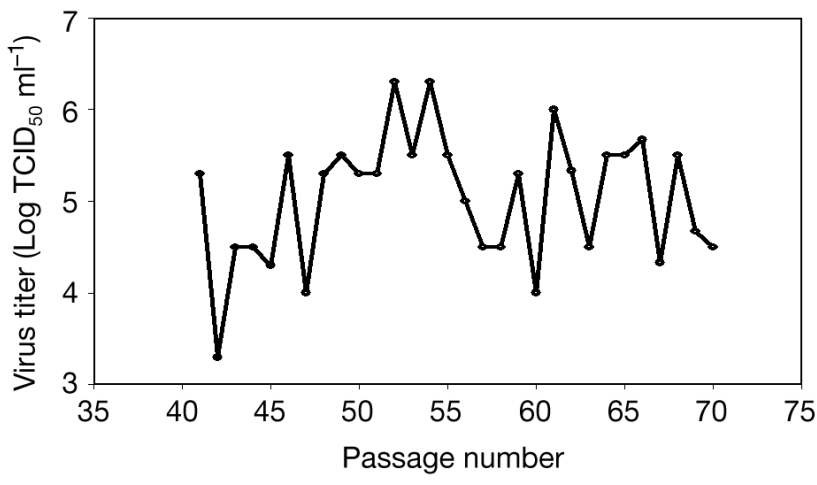

Fig. 5. Fluctuation of BBNNV titers in the supernatants of BB cells from Subcultures 41 to 70 . Each supernatant was collected from BB cells $5 \mathrm{~d}$ post-subculture

temperatures. The differences among these viral titers were less than $1.3 \log \mathrm{TCID}_{50} \mathrm{ml}^{-1}$, indicating that virus production in the BB cell line at these 4 different temperatures had similar sensitivity. This result suggested that temperature-sensitive (ts) mutants were unlikely to be the cause of NNV persistence in the BB cell line.

\section{Detection of DI particles in BBNNV}

During the purification of BBNNV and B00GD, only 1 band with a buoyant density of $1.34 \mathrm{~g} \mathrm{ml}^{-1}$ was formed in the $\mathrm{CsCl}$ gradient for both viruses. Genomic RNAs were extracted from both purified viruses and analyzed using a formaldehyde gel. No smaller RNA1 or RNA2 was found in the purified BBNNV or B00GD (Fig. 6). Furthermore, the size of the RNA1 or RNA2 of BBNNV was the same as that of B00GD.

The protein profiles of the purified BBNNV and B00GD were analyzed by SDS-PAGE and Western blot using GNNV-specific MAb 9D. The molecular weight of the coat protein of BBNNV was a little lower than that of B00GD (Fig. 7). However, in both cases, only a single band of the coat protein was observed, and no other smaller bands were present.

\section{Comparison of the deduced amino acids between BBNNV and 9 other Taiwan NNV isolates}

The identities of the deduced amino acids (aa) of the T2 region (aa 54 to 331) for BBNNV and the 9 reported Taiwan NNV isolates were $>99 \%$. However, differences were observed at 3 positions (aa 61, 130, 240); however, these did not cause obvious changes in the antigenic indexes of the deduced amino acid sequences of the T2 region of BBNNV compared to the 9 other Taiwan NNV isolates (Table 1). 


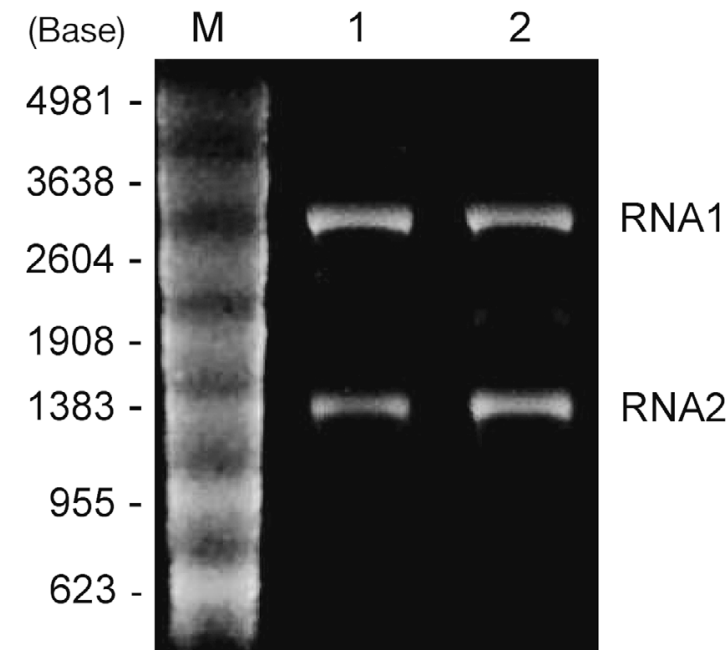

Fig. 6. Electrophoresis of genomic RNAs extracted from the purified B00GD particles (Lane 1) and BBNNV particles (Lane 2). M: RNA marker (Promega)

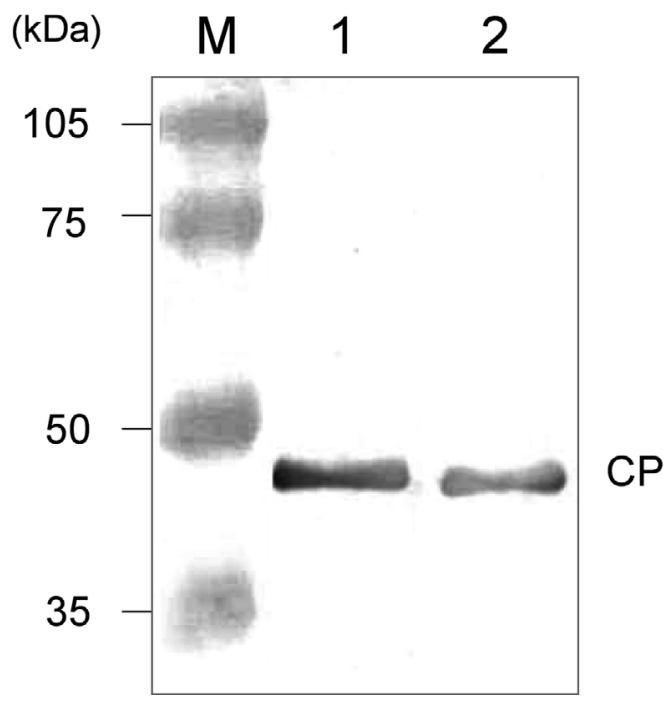

Fig. 7. Western blot analysis of polypeptide profiles of purified B00GD (Lane 1) and BBNNV (Lane 2) obtained using GNNV coat protein-specific MAb 9D. M: protein marker; CP: coat protein

Table 1. Comparison of the 3 different deduced amino acids between BBNNV and the 9 other Taiwan NNV isolates. The 9 Taiwan NNV isolates are described in 'Materials and methods'. AI: antigenic index, a computer algorithm for predicting the topological feature of a protein from its primary amino acid sequence

\begin{tabular}{|c|c|c|c|c|}
\hline \multirow{2}{*}{$\begin{array}{l}\text { Amino } \\
\text { acid site }\end{array}$} & \multicolumn{2}{|c|}{$-\mathrm{BBNNV}-$} & \multicolumn{2}{|c|}{ — Taiwan NNV isolates - } \\
\hline & Amino acid type & AI & Amino acid type & AI \\
\hline aа 61 & Alanine (A) & 0.3 & Serine $(\mathrm{S})$ & 0.3 \\
\hline aа 130 & Asparagine (N) & 1.6 & Aspartic acid (D) & 2.2 \\
\hline aа 240 & Threonine (T) & -0.3 & Serine $(\mathrm{S})$ & 0.6 \\
\hline
\end{tabular}

Table 2. Comparison of the neutralization indexes (NI) between BBNNV and B00GD using 5 neutralizing MAbs against GNNV (G9508KS). Values are $\log _{10}$ NI (where NI is the viral titer before MAb treatment/the viral titer after MAb treatment). The values above 1.7 are considered significant, with good neutralization

\begin{tabular}{|lccc|}
\hline MAbs & BBNNV & B00GD & $\begin{array}{c}\text { Ratio } \\
\text { (BBNNV/B00GD) }\end{array}$ \\
\hline 2E & 4.0 & 3.4 & 1.2 \\
9B & 4.5 & 5.2 & 0.9 \\
9D & 4.5 & 4.9 & 0.9 \\
10G & 3.5 & 4.1 & 0.9 \\
12E & 5.5 & 5.1 & 1.1 \\
\hline
\end{tabular}

Five neutralizing MAbs against GNNV were used for the comparison of neutralization indexes between BBNNV and B00GD. All of the 5 MAbs were able to neutralize BBNNV as well as B00GD, and the values of $\log N I$ between BBNNV and B00GD were similar (Table 2).

\section{DISCUSSION}

In the present study, a novel cell line (BB) was developed from the brain tissue of Barramundi Lates calcarifer that had survived VNN disease. The BB cell line was found to have NNV persistence, because culture supernatant from the BB cells was able to induce a complete CPE of GF-1 cells, and both NNV nucleic acid and coat protein were detected in the BB cells. This is the first reported cell line that has been persistently infected with NNV. Moreover, this cell line was established directly from a survivor fish of VNN disease, and not from artificial infection of a normal cell line with NNV. Accordingly, the BB cell line will become an important model for realizing the mechanism of NNV-persistent infection.

There are at least 3 entities proposed for the maintenance of viral persistence in cell cultures - temperature mutants, DI particles and interferon (Joklik 1977, Hedrick \& Fryer 1981). Persistent infection may also result from a combination of these events. This study examined the first 2 possibilities.

Temperature-sensitive (ts) mutants have a conditional-lethal phenotype. The viral protein is able to assume a functional configuration at a permissive temperature, and allows the ts mutant to propagate (Fields et al. 1996). If ts mutants were the major cause of NNV persistence in the $\mathrm{BB}$ cell line, the production of BBNNV would change drastically with temperature variation $\left(20\right.$ to $\left.30^{\circ} \mathrm{C}\right)$. This, however, was not the case in BB cells. 
Homologous interference, which occurs within the cell and is only exhibited against a homologous virus or closely related viruses, has been noted with animal viruses (Fields et al. 1996). It usually occurs in viruses that have been serially passaged at high multiplicity (Hedrick \& Fryer 1981). Macdonald \& Yamamoto (1978) stated that infectious pancreatic necrosis virus (IPNV) exhibits autointerference linked to the presence of an interfering virus particle. The interfering factor was co-purified with the standard virus, but enriched in the region immediately above the standard virus in a shallow $\mathrm{CsCl}$ gradient, indicating that the factor was distinct from the standard virus; it was also proven to be defective in replication by interfering ability assay. Lo et al. (1991) examined the interfering virus population of IPNV and showed that a proportion of the large viral genomes had deleted genome segments. The deletion mutants may compete with the standard viruses for components of the replication apparatus, and give rise to an increasing proportion of DI particles.

Since only $1 \%$ of the BB cells released viral particles, it would be difficult to purify BBNNV by $\mathrm{CsCl}$ gradient centrifugation, even using a large-scale culture of BB cells. If DI particles were present, the use of undiluted culture supernatants of BB cells would ensure that the ratio of DI particles and the standard viruses remain unchanged. Therefore, we used undiluted culture supernatant of BB cells to infect GF-1 cells, in order to get enough viral particles for the following analysis. If DI particles were abundant in the BBNNV populations, there would be 2 bands formed after $\mathrm{CsCl}$ gradient centrifugation. Yet, only 1 band was observed in this study. Moreover, neither a smaller genome segment of RNA1 or RNA2, nor a smaller viral coat protein was found in the purified BBNNV particles (Figs. $6 \&$ 7). In addition, the molecular weights of genomic RNA1 and RNA2 of BBNNV were identical to those of B00GD. Although deletion of only a very few nucleotides/ amino acids is still possible, as this will not noticeably affect RNA/protein migration during electrophoresis, the number of nucleotides in the $\mathrm{T} 2$ region $(85.5 \%$ of the open reading frame of the coat protein gene) of BBNNV is found to be the same as that of B00GD. Although the mobility of the coat protein of BBNNV in SDS-PAGE ran a little faster than that of B00GD, it is possible that this was due to the lower level of glycosylation or phosphorylation of BBNNV, or to the minor differences in charges carried by the coat proteins between the 2 viruses. Furthermore, autointerference was never observed during titration of BBNNV from the culture supernatants of the BB cells, and complete CPE could always be induced in GF-1 cells by inoculation of the undiluted BB culture supernatants (Fig. 2). Therefore, DI particles are unlikely to be responsible for the maintenance of NNV-persistent infection in the BB cell line.

If a lytic virus is going to establish a persistent infection in a susceptible host, alternative virus-host cell interactions must occur to limit the CPE of the viruses, and the virus will co-evolve with the host cells. Since the immune response is specific for a particular antigenic determinant, the virus may reduce the efficiency of the immune response by altering the structure of antigenic determinants in its structural proteins. Therefore, we compared the deduced amino acid sequence of the $\mathrm{T} 2$ region of the coat protein gene of BBNNV with those of the 9 other Taiwan NNV isolates. We also compared the neutralization indexes of GNNV-specific MAbs against BBNNV and B00GD, which were isolated from barramundi larvae during the acute stage of VNN disease. The alignment results revealed that 3 amino acids (aa 61, 130 and 240) of BBNNV were consistently different from those of the 9 other Taiwan NNV isolates. These 3 amino acids are changed from uncharged polar serine (S) to non-polar alanine (A) at aa 61, from charged acidic polar aspartic acid (D) to uncharged polar asparagine $(\mathrm{N})$ at aa 130 and from uncharged polar serine $(\mathrm{S})$ to threonine $(\mathrm{T})$ at aa 240 for BBNNV. However, the changes of these 3 amino acids did not make an obvious difference in the antigenic indexes of the T2 region of the coat protein gene. In addition, the result of the neutralization test, using 5 neutralizing MAbs against GNNV, revealed that the antigenic determinants present on B00GD also existed on BBNNV.

In persistent viral infection of cultured cells, the virus may be released from all cells at a low level, or from only a small fraction of the total cells. In the latter situation, the few infected cells release lytic viruses and are killed, but the progeny viruses go on to infect only a small number of the total cells, while the majority of the cells remains uninfected. From the results of immunostaining revealed in Fig. 4, the persistent infection of the BB cell line belongs to this category. This restriction could be due to the availability of only a limited number of susceptible cells at a given time, or due to the presence of soluble inhibitors such as interferon in the culture medium (Sekellick \& Martin 1978, Fields et al. 1996). Although there were different morphologies of cells in the primary culture, only 1 type of cells became dominant and remained consistent in the continuous BB cell line. All BB cells reacted with only 1 of the 3 MAbs against different cell markers, thus indicating that the BB cell line consists of 1 cell type. Therefore, the reason for only a small fraction of total BB cells being infected with BBNNV is unlikely due to only a few cells susceptible to BBNNV.

It is still unclear why BB cells reacted with MAbs that are against a broad spectrum of keratin in epithelial 
tumors. It is suspected that epithelial cells were derived from the pia mater that covered the brain. When the brain was removed from the fish, it is very possible that the pia mater, the innermost part of the meninges, was also removed along with the brain. The pia mater is a thin layer of squamous epithelial cells composed of modified fibroblasts. After serial subcultures, primary culture gradually becomes a continuous cell line. With each successive subculture, that component of the population with the ability to proliferate most rapidly will gradually predominate, and nonproliferating or slowly proliferating cells will be diluted out. Cultures from tissues that renew only under stress (e.g. muscle, glia) may only contain committed precursor cells with a limited life span in vitro; thus, the identity of the cultured cell is not only defined by its lineage (e.g. brain) in vivo (Freshney 1994).

Thus, the interferon appears to best explain the persistence of NNV infection in the BB cell line; this line of research will be carefully explored in a series of experiments presently being pursued in our laboratory.

Acknowledgements. The authors would like to thank Miss S. J. Yu for helpful criticism of the manuscript and the National Science Council of the Republic of China for financially supporting this research under Contract No. NSC-91-2311-B002-058.

\section{LITERATURE CITED}

Chi SC, Lo CF, Kou GH, Chang PS, Peng SE, Chen SN (1997) Mass mortalities associated viral nervous necrosis disease in two species of hatchery-reared grouper, Epinephelus fuscogutatus and Epinephelus akaara (Temminck \& Schlegel). J Fish Dis 20:185-193

Chi SC, Hu WW, Lo BJ (1999) Establishment and characterization of a continuous cell line (GF-1) derived from grouper, Epinephelus coioides (Hamilton): a cell line susceptible to grouper nervous necrosis virus (GNNV). J Fish Dis 22:173-182

Chi SC, Lo BJ, Lin SC (2001a) Characterization of grouper nervous necrosis virus (GNNV). J Fish Dis 24:3-13

Chi SC, Shieh JR, Shieh YL, Chang JK (2001b) Development, characterization and application of monoclonal antibodies against grouper nervous necrosis virus (GNNV). In: Proceedings of the 10th international conference on disease of fish and shellfish (abstract), 9-14 September, Dublin. European Association of Fish Pathologists, Dublin, p 14

Chi SC, Shieh JR, Lin SJ (2003) Genetic and antigenic analysis of betanodaviruses isolated from aquatic organisms in Taiwan. Dis Aquat Org 55: 221-228

Comps M, Pepin JF, Bonami JR (1994) Purification and characterization of two fish encephalitis viruses (FEV) infecting Lates calcarifer and Dicentrarchus labrax. Aquaculture 123:1-10

Editorial responsibility: Jo-Ann Leong,

Kaneohe, Hawaii, USA
Dimmock NJ, Primrose SB (1994) Introduction to modern virology, 4th edn. Blackwell Science, Malden, MA

Fields BN, Dnipe DM, Howley PM (1996) Fundamental virology, 3rd edn. Lippincott, Williams \& Wilkins, Philadelphia, PA

Freshney RI (1994) Culture of animal cells - a manual of basic technique, 3rd edn. Wiley-Liss, New York

Hedrick RP, Fryer JL (1981) Persistent infection of three salmonid cell lines with infectious pancreatic necrosis virus (IPNV). Fish Pathol 15:163-172

Hedrick RP, Leong JC, Fryer JL (1978) Persistent infections in salmonid fish cells with infectious pancreatic necrosis virus (IPNV). J Fish Dis 1:297-308

Jameson BA, Wolf H (1988) The antigenic index: a novel algorithm for predicting antigenic determinants. Comput Appl Biosci 4(1):181-186

Johansen R, Amundsen M, Dannevig BH, Sommer AI (2003) Acute and persistent experimental nodavirus infection in spotted wolffish Anarhichas minor. Dis Aquat Org $57: 35-41$

Joklik WK (1977) Mechanisms of establishment and maintenance of persistent infections. In: Schlessinger D (ed) Microbiology. American Society of Microbiology, Washington, DC, p 434-438

Lee KW, Chi SC, Cheng TM (2002) Interference of the life cycle of fish nodavirus with fish retrovirus. J Gen Virol 83: 2469-2474

Lo CF, Wang CH, Lin MS, Kou GH (1991) Viral peptides and RNA of defective interfering particles of IPNV virus generated by serial undiluted passaging in TO-2 cells. Fish Pathol 26:119-125

Macdonald RD, Yamamoto T (1978) Quantitative analysis of defective interfering particles in infectious pancreatic necrosis virus preparations. Arch Virol 57:77-89

Mahy BWJ, Kangro HO (1996) Neutralization. In: Mahy BWJ, Kangro HO (eds) Virology method manual. Academic Press, London, p 108-109

Mori K, Nakai T, Muroga K, Arimoto M, Mushiake K, Furusawa I (1992) Properties of a new virus belonging to Nodaviridae found in larval striped jack (Pseudocaranx dentex) with nervous necrosis. Virology 187:368-371

Munday BL, Kwang J, Moody N (2002) Betanodavirus infection of teleost fish: a review. J Fish Dis 25:127-142

Nishizawa T, Mori K, Nakai T, Furusawa I, Muroga K (1994) Polymerase chain reaction amplification of RNA of striped jack nervous necrosis virus (SJNNV). Dis Aquat Org 18: 103-107

Nishizawa T, Furuhashi M, Nagal T, Nakai T, Muroga K (1997) Genomic classification of fish nodaviruses by molecular phylogenetic analysis of the coat protein gene. Appl Environ Microbiol 63:1633-1636

Rima BK, Martin SJ (1976) Persistent infection of tissue cultured cells by RNA viruses. Med Microbiol Immunol 162: 89-118

Sekellick MJ, Martin SJ (1978) Persistent infection. I. Interferon-inducing defective interfering particles as mediators of cell sparing: possible role in persistent infection by vesicular stomatitis virus. Virology 85:175-186

Shieh JR, Chi SC (2005) Production of monoclonal antibodies against grouper nervous necrosis virus (GNNV) and development of an antigen capture ELISA. Dis Aquat Org 63:53-60

Submitted: July 21, 2004; Accepted: March 17, 2005

Proofs received from author(s): June 20, 2005 